\title{
Infected Dermoid Cyst of the Posterior Fossa: Case Report
}

\author{
Marouane Hammoud* \\ Department of Neurosurgery, Hassan II University Hospital of Fez, Morocco
}

*Corresponding author: Medicine doctor, Department of Neurosurgery, Hassan II

University Hospital of Fez, University Sidi Mohammed Ben Abdellah, Fez, Morocco.

Received Date: October 07, 2021

Published Date: October 28, 2021

\section{Introduction}

Intracranial dermoid cysts are a rare congenital benign neoplasms, they account for only $0.1 \%-0.7 \%$ of all intracranial tumors [1]. They arise from inclusion of ectodermal elements within the neural tube during its closing between the third and fifth week of embryonic development [2]. Patients with a posterior fossa dermoid cyst and an associated dermal sinus may develop bacterial meningitis or abscess formation of the dermoid itself [3]. Here in, we present a case of dermoid cyst with occipital dermal sinus, complicated by abcess.

\section{Case Report}

A 5-year-old girl with an unremarkable medical history was admitted to pediatric unit for 1 month history of occipital headache, and 2 weeks of neck stiffnes. Physical examination revealed ataxia, a temperature of $38.1^{\circ} \mathrm{C}$, and also a small dermal orifice in the midline posterior fossa without pus. The result of routine blood examination was normal CT scan showed a large cystic mass in vermis, higher attenuation on CT than CSF, without contrast enhancement.

The diagnosis of dermoid cyst was suspected, the patient underwent a total excision, through a suboccipital approach, after opening the dura, we found an encapsulated cystic mass with hair and purulence contents. Pathologic examination revealed a dermoid cyst The culture of the discharging material revealed Staphylococcus epidermidis.

Followed by systemic antibiotic therapy with a good outcome (Figure $1 \& 2$ ).

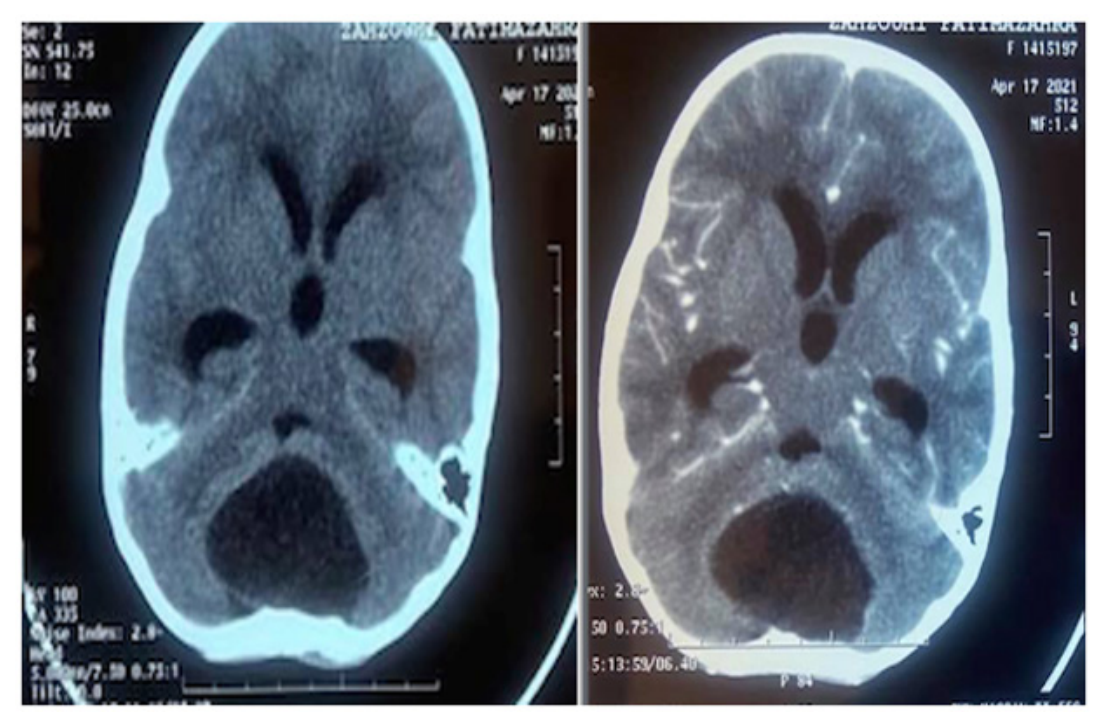

Figure 1: CT scan showing a cytic lesion of the posterior fossa (on the left) without enhancement after injection (On the right). 


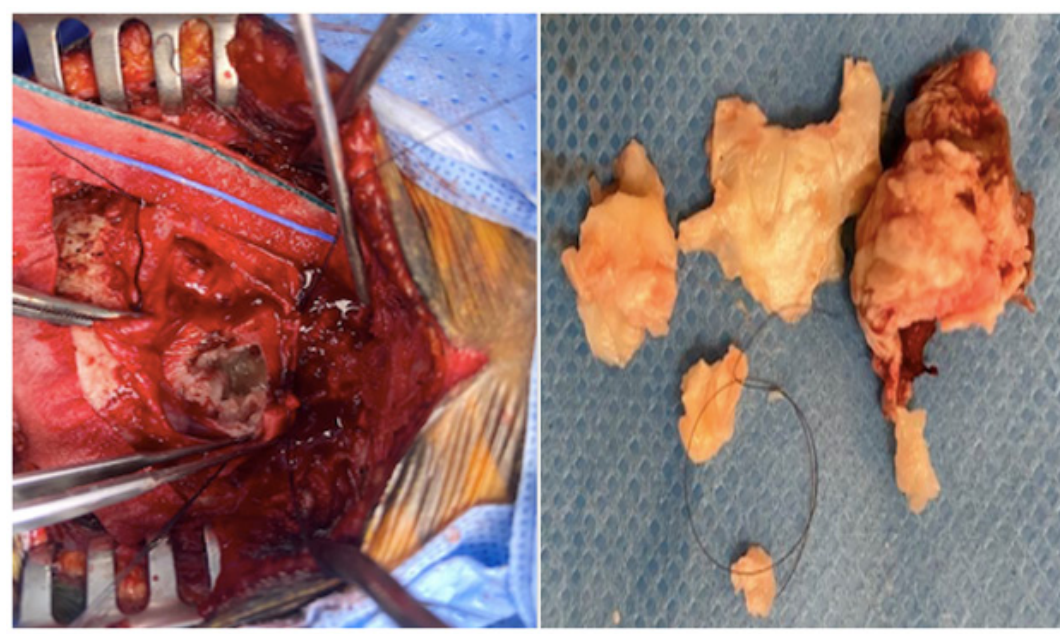

Figure 2: On the left Intra-operation photo showing the purulence content. On the left, image showing the removed contents

\section{Discussion}

Intracranial dermoid cyst is a congenital benign neoplasm that grows slowly as a result of progressive epithelial desquamation and gland secretion within the cyst [4]. It accounts for $0.1 \%-0.7 \%$ of all intracranial tumors, they mostly occur in the posterior fossa, particularly in the midline position in the vermis or adjacent meninges [3]. The development of those lesions is associated with a defect of closure of the neural tube in the third to fourfth week of embryonic life frenquently associated with dermal lesion, A neurocutaneous fistula can be the portal entry, micro-organisms may travel into the cyst with potential risk of deeper suppurations. The clinical presentation may be increased intracranial pressure, cerebellar syndromes, or meningitis sometimes a bone defect may be palpable $[5,6]$.

The pre- operative diagnosis is possible by using CT scan and MRI. CT scan shows a low-density mass with values of fat present in these tumors [7], the surrinfection of the cyst causes enhancement. Microsurgical resection of the tumor is the treatment of choice for dermoid cysts [4,5]. Total removal was not always performed because of the firm adhesion of the tumor capsule concerning cerebellar abscesses, the choice of treatment depends on the clinical status of the patient, as well as on the size, location, and the presence or absence of the capsule [5,8].

There have been very few publications about the long- term follow-up of dermoid cyst patientsm No recurrence of a posterior fossa dermoid cyst after surgery has been reported in the literature. Hashmi et al. reported the reappearance of a cerebellar abscess 20 years after ex- cision of a dermoid cyst [9]. In our case the 2 years follow-up showed no reccurence.

\section{Conclusion}

Dermoid cyst of the posterior fossa should be considered in all children with occipital skin lesion. Early surgical treatment is necessary to prevent bacterial infection through the dermal sinus.

\section{Acknowledgement}

None.

\section{Conflict of Interest}

No conflict of interest.

\section{References}

1. Lunardi P, Missori P, Gagliardi FM, Fortuna A (1990) Dermoid cysts of the posterior cranial fossa in children. Report of nine cases and review of the literature. Surg Neurol 34(1): 39-42.

2. Hsu ST, Yu-Yun Lee J, Chao SC, Hsieh MY, Huang CC (1998) Congenital occipital dermal sinus with intracranial dermoid cyst complicated by recurrent Escherichia coli meningitis. Br J Dermatol 139: 922-924.

3. Akhadar A, Jiddane M, Chakir N, Rachid El Hassani, Brahim Moustarchid, et al. (2002) Cere- bellar abscesses secondary to occipital dermoid cyst with dermal sinus. Surg Neurol 58: 266-270.

4. Guidetti B, Gagliardi FM (1977) Epidermoid and dermoid cysts. Clinical evaluation and late surgical results. J Neurosurg 47: 12-18.

5. Hayek G, Mercier Ph, Fournier HD, Menei Ph, Pou- plard D, et al. (2001) Sinus dermique avec kyste dermoide de la fosse cére'brale poste'rieure réve'le' par un abce`s. A propos de deux cas pédiatriques avec revue de la litte'rature. Neurochir 47: 123-127.

6. Martinez-Lage JF, Ramos J, Puche A, Poza M (1997) Extra- dural dermoid tumours of the posterior fossa. Arch Dis Child 77: 427-430.

7. Brown JY, Morokoff AP, Mitchell PJ, Gonza- les MF (2001) Unusual imaging appearance of an in- tracranial dermoid cyst. AJNR Am J Neuroradiol 22: 1970-197.

8. Schijman E, Monges J, Cragnaz R (1986) Congenital dermal sinuses, dermoid and epidermoid cysts of the posterior fossa. Childs Nerv Syst 2: 83-86.

9. Hashmi S, Jones R (1998) Delayed recurrence of cerebellar abscess 20 years after excision of dermoid cyst and sinus. Br J Neurosurg 12: 358360 